\title{
Stability of Spreading Processes over Time-Varying Large-Scale Networks
}

\author{
Masaki Ogura, Member, IEEE, and Victor M. Preciado, Member, IEEE
}

\begin{abstract}
In this paper, we analyze the dynamics of spreading processes taking place over time-varying networks. A common approach to model time-varying networks is via Markovian random graph processes. This modeling approach presents the following limitation: Markovian random graphs can only replicate switching patterns with exponential inter-switching times, while in real applications these times are usually far from exponential. In this paper, we introduce a flexible and tractable extended family of processes able to replicate, with arbitrary accuracy, any distribution of inter-switching times. We then study the stability of spreading processes in this extended family. We first show that a direct analysis based on Itô's formula provides stability conditions in terms of the eigenvalues of a matrix whose size grows exponentially with the number of edges. To overcome this limitation, we derive alternative stability conditions involving the eigenvalues of a matrix whose size grows linearly with the number of nodes. Based on our results, we also show that heuristics based on aggregated static networks approximate the epidemic threshold more accurately as the number of nodes grows, or the temporal volatility of the random graph process is reduced. Finally, we illustrate our findings via numerical simulations.
\end{abstract}

Index Terms-Dynamic random graphs, complex networks, epidemics, stochastic processes, random matrix theory.

\section{INTRODUCTION}

U NDERSTANDING spreading processes in complex networks is a central question in the field of Network Science with applications in rumor propagation [1], malware spreading [2], epidemiology, and public health [3]. In this direction, important advances in the analysis of spreading processes over time-invariant (TI) networks have been made during the last decade (see $[4]$ and $[5]$ for recent surveys). Under the time-invariance assumption, we find several recent breakthroughs in the literature, such as the rigorous analysis of mean-field approximations [6], the connection between the eigenvalues of a network and epidemic thresholds [7], [8], [9], new modeling tools for analysis of multilayer networks $|10|$, and the use of control and optimization tools to contain epidemic outbreaks [11], [12], [13], [14].

In practice, most spreading processes of practical interest take place in networks with time-varying (TV) topologies, such as human contact networks, online social networks, biological, and ecological networks [15]. A naive, but common, approach to analyze dynamic processes in TV networks is to build an aggregated static topology based on time averages. When the time scales of the network evolution are comparable to those of the spreading process, the static aggregated graph is not well-suited to study the dynamics of TV networks, as pointed out in [16], [17]. Using extensive numerical simulations, it has been observed that the speed of spreading of a disease in a TV network can be substantially slower than in its aggregated static representation [18]. This observation is also supported by the study of spreading processes in a real TV network constructed from a mobile phone dataset [19]. More recent studies point

- The authors are with the Department of Electrical and Systems Engineering at the University of Pennsylvania, Philadelphia, PA 19104. out the key role played by the addition and removal of links [20], as well as the distribution of contact durations between nodes |21| in the dynamics of the spread. The works mentioned above provide empirical evidence about the nontrivial effect that the dynamics of the network has on the behavior of spreading processes.

Apart from these empirical studies, we also find several works providing theoretical support to these numerical observations. The authors in [22], [23] derived the value of the epidemic threshold in particular types of TV networks, assuming that all nodes in the network present a homogeneous infection and recovery rates. In [24], Perra et al. proposed a model of temporal network, called the activitydriven model, able to replicate burstiness in node/edge activity and analyzed contagion processes taking place in this model in [25]. Karsai et al. proposed in [26] a timevarying network using a reinforcement process able to replicate the emergence of strong and weak ties observed in a mobile call dataset. A wide and flexible class of TV network model, called edge-Markovian graphs, was proposed in [27] and analyzed in [28]. In this model, edges appear and disappear independently of each other according to Markov processes. Edge-Markovian graphs have been used to model, for example, intermittently-connected mobile networks [29], [30] and information spread thereon [31]. Taylor et al. derived in [32] the value of the epidemic threshold in edge-Markovian graphs and proposed control strategies to contain an epidemic outbreak, assuming homogeneous spreading and recovery rates.

It is worth remarking that, although most existing analyses of spreading processes over dynamic networks rely on the assumption that nodes present homogeneous rates and the Markov processes used to generate edge-switching signals are identical, these assumptions are not satisfied in realworld networks. Furthermore, most theoretical analyses 
mentioned above assume that edges appear and disappear in the network according to exponential distributions. However, as was found in several experimental studies [33], [34], [35] utilizing radio-frequency identification devices (RFID), the probability distribution of contact durations in human proximity networks is far from exponential. Therefore, it is of practical interest to develop tools to analyze spreading processes in TV networks with heterogenous rates and non-identical edge-switching signals able to replicate nonexponential switching patterns.

In this paper, we study spreading processes over TV networks with heterogeneous rates and non-identical edgeswitching signals. First, we propose a wide class of TV networks where edges appear and disappear following aggregated Markov processes [36]. Since the class of aggregated Markov processes contains all Markov processes, the proposed class includes all edge-Markovian graphs [27]. We furthermore observe that, due to the modeling flexibility of aggregated Markov processes, the proposed class of TV networks can replicate human-contact durations following non-exponential distributions with an arbitrary accuracy. Second, we theoretically analyze the dynamics of spreading process over the proposed class of dynamic graphs. We model the dynamics of spreading processes on this class using a set of stochastic differential equations, which is a timevarying version of the popular $N$-intertwined SIS model [9]. Third, we derive conditions for these stochastic differential equations to be globally exponentially stable around the infection-free equilibrium, i.e., the infection 'dies out' exponentially fast over time. One of the main challenges in this analysis is to derive computationally tractable stability conditions. For example, as will be illustrated later, if we simply apply a recently proposed stability condition [37] based on the direct application of Itô's formula for jump processes (see, e.g., [38|), we obtain a stability condition that requires the computation of the largest eigenvalue of a matrix whose size grows exponentially with the number of edges in the network. Hence, this condition is hard (if not impossible) to verify for large-scale networks. In this paper, we use spectral graph theory [39] to derive stability conditions for the set of stochastic difference equations in terms of the largest eigenvalues of a matrix whose size grows linearly with the number of nodes in the network.

This paper is organized as follows. In Section 2, we introduce the notation and preliminary facts used in the paper and, then, introduce a flexible model of TV networks model called aggregated-Markovian random graph process. In Section 3 , we state computationally tractable stability conditions for spreading processes taking place in this dynamic network model. In Section 4 , we illustrate our results with some numerical examples. The proofs of the theorems are presented in Section 5

\section{Preliminaries and Problem Statement}

This section starts by introducing notation in Subsection 2.1 Then, in Subsection 2.2. we review preliminary facts about spreading processes over static networks. In Subsection 2.3 we introduce the class of TV network models under consideration. We finalize this section by stating the problem to be studied in Subsection 2.4

\subsection{Notation}

For a positive integer $n$, define the set $[n]=\{1, \ldots, n\}$. For $c \in \mathbb{R}$, define $c^{-}=(|c|-c) / 2$. Let $\operatorname{sgn}(\cdot)$ denote the sign function over $\mathbb{R}$, which can be extended to matrices by entry-wise application. The Euclidean norm of $x \in \mathbb{R}^{n}$ is denoted by $\|x\|$. Let $I_{n}$ denote the $n \times n$ identity matrix. A square matrix is said to be Metzler if its off-diagonal entries are nonnegative. The spectral abscissa of a square matrix $A$, denoted by $\eta(A)$, is defined as the maximum real part of its eigenvalues. We say that $A$ is Hurwitz stable if $\eta(A)<0$. Also, we define the matrix measure [40] of $A$ by $\mu(A)=\eta\left(A+A^{\top}\right) / 2$. For a square random matrix $X$, its expectation is denoted by $E[X]$ and its variance is defined by $\operatorname{Var}(X)=E\left[(X-E[X])^{2}\right]$. The diagonal matrix with diagonal elements $a_{1}, \ldots, a_{n}$ is denoted by $\operatorname{diag}\left(a_{1}, \ldots, a_{n}\right)$.

A directed graph is defined as the pair $\mathcal{G}=(\mathcal{V}, \mathcal{E})$, where $\mathcal{V}=\left\{v_{1}, \ldots, v_{n}\right\}$ is a set of nodes ${ }^{1}$ and $\mathcal{E} \subseteq \mathcal{V} \times \mathcal{V}$ is a set of directed edges (also called diedges or arcs), defined as ordered pairs of nodes. By convention, we say that $\left(v_{j}, v_{i}\right)$ is a directed edge from $v_{j}$ pointing towards $v_{i}$. The adjacency matrix $A=\left[a_{i j}\right]$ of a directed graph $\mathcal{G}$ is defined as the $n \times n$ matrix such that $a_{i j}=1$ if $\left(v_{j}, v_{i}\right) \in \mathcal{E}$, and $a_{i j}=0$ otherwise.

A stochastic process $\sigma$ taking values in a set $\Gamma$ is said to be an aggregated Markov process (see, e.g., [36]) if there exists a time-homogeneous Markov process $\theta$ taking values in a set $\Lambda$ and a function $f: \Lambda \rightarrow \Gamma$ such that $\sigma=f(\theta)$. Throughout the paper, it is assumed that $\Lambda$ is finite, $f$ is surjective, and all the Markov processes are time-homogeneous. We say that a Markov process $\theta$ is irreducible if all the states in $\Lambda$ can be reached from any other state in $\Lambda$ (see, e.g., [41] for more details). We say that an aggregated Markov process $\sigma=f(\theta)$ is irreducible if $\theta$ is irreducible. We will use the next lemma in our proofs.

Lemma 2.1 ([41]). Let $\theta$ be an irreducible Markov process taking values in a finite set $\Lambda$. Then, $\theta$ has a unique stationary distribution $\pi$. Moreover,

$$
\pi(\lambda)=\lim _{t \rightarrow \infty} \operatorname{Pr}(\theta(t)=\lambda),
$$

for every $\lambda \in \Lambda$ and $\theta(0) \in \Lambda$.

\subsection{Spreading Process over Static Networks}

In this subsection, we review a model of spreading processes over static networks called the Heterogeneous Networked SIS (HeNeSIS) model, which is an extension of the popular $N$-intertwined SIS model [9] to the case of nodes with heterogeneous rates. This model can be described using a continuous-time Markov process, as follows. Let $\mathcal{G}$ be a directed graph, where nodes in $\mathcal{G}$ represent individuals and diedges represent interactions between them (which we consider to be directed). At a given time $t \geq 0$, each node can be in one of two possible states: susceptible or infected. We define the variable $X_{i}(t)$ as $X_{i}(t)=1$ if node $i$ is infected at time $t$, and $X_{i}(t)=0$ if $i$ is susceptible. In the HeNeSIS model, when a node $i$ is infected, it can randomly transition to the susceptible state according to a Poisson process with rate $\delta_{i}>0$, called the recovery rate of node $i$.

1. For simplicity in notation, we sometimes refer to node $v_{i}$ as $i$. 
On the other hand, if node $i$ is in the susceptible state, it can randomly transition to the infected state according to a Poisson process with rate $\beta_{i} \sum_{i=1}^{n} a_{i j} X_{j}(t)$, where $\beta_{i}>0$ is called the infection rate of node $i$. In other words, the rate of transition of node $i$ from susceptible to infected is proportional to the number of its infected neighbors.

Since the above Markov process has a total of $2^{n}$ possible states (two states per node), its analysis is very hard for arbitrary contact networks of large size. A popular approach to simplify the analysis of this type of Markov process is to consider upper-bounding linear models. Let $A$ denote the adjacency matrix of $\mathcal{G}$, and define $p=\left(p_{1}, \ldots, p_{n}\right)^{\top}$, $P=\operatorname{diag}\left(p_{1}, \ldots, p_{n}\right), B=\operatorname{diag}\left(\beta_{1}, \ldots, \beta_{n}\right)$, and $D=$ $\operatorname{diag}\left(\delta_{1}, \ldots, \delta_{n}\right)$. Then, it is known that the solutions $p_{i}(t)$ $(i=1, \ldots, n)$ of the linear differential equation

$$
\dot{p}=(B A-D) p
$$

upper-bounds the evolution of $E\left[X_{i}(t)\right](i=1, \ldots, n)$ from the exact Markov process with $2^{n}$ states [9]. Thus, if the dynamics in (1) is globally exponentially stable, the infection dies out exponentially fast in the exact Markov process [6], [13|. In the case of homogeneous infection and recovery rates, i.e., $\beta_{i}=\beta$ and $\delta_{i}=\delta$ for all $i$, the dynamics (1) is globally exponentially stable if and only if $|9|$

$$
\frac{\beta}{\delta}<\frac{1}{\eta(A)} .
$$

Apart from the continuous-time Markov process described above, we can also model the dynamics of a spread using a discrete-time networked Markov chain with an exponential number of states $[\overline{8}]$. As in the continuoustime case, we can show $[8]$ that the solution of the linear difference equation

$$
p(k+1)=(B A+I-D) p(k),
$$

where $p=\left(p_{1}, \ldots, p_{n}\right)^{\top}$, upper-bounds the probabilities $\operatorname{Pr}(i$ is infected at time $k)(i=1, \ldots, n)$. As in the continuous-time case, it turns out that if the discrete-time linear system in (3) is globally exponentially stable, the infection dies out exponentially fast in the exact Markov process $|6|$. In the case of homogeneous infection and recovery rates, the system in (3) is stable if and only if (2) holds [8].

\subsection{Aggregated-Markovian Random Graph Processes}

In this subsection, we introduce two new models of TV networks: the aggregated-Markovian edge-independent (AMEI) and the aggregated-Markovian arc-independent (AMAI) models. As we mentioned above, these models generalize the class of edge-Markovian time-varying networks [27]. Let $\mathcal{G}=\{\mathcal{G}(t)\}_{t \geq 0}$ be a stochastic graph process taking values in the set of directed graphs with $n$ nodes. Let $A(t)$ denote the adjacency matrix of $\mathcal{G}(t)$ for each $t \geq 0$. The class of dynamic random graphs studied in this paper is defined as follows.

Definition 2.2. Consider a collection of aggregated Markov processes $\sigma_{i j}=g_{i j}\left(\theta_{i j}\right)$ where $g_{i j}$ are $\{0,1\}$-valued functions and $\theta_{i j}$ are stochastically independent Markov processes for $1 \leq i<j \leq n$. An aggregated-Markovian

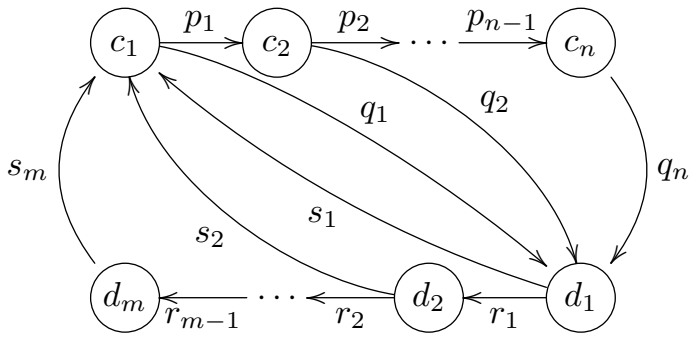

Fig. 1. A Markov process described in Example 2.3

edge-independent (AMEI) network is a random graph process in which $A_{i j}=A_{j i}=\sigma_{i j}$ for $i<j$ and $A_{i i}=0$ for all $i \in[n]$. If $\sigma_{i j}$ is irreducible for all possible pairs $(i, j)$, then we say that $\mathcal{G}$ is irreducible.

In other words, the upper-triangular entries of the timevarying adjacency matrix $A(t)$ of an AMEI graph are independent aggregated Markov processes. The lower triangular entries are constructed via symmetry and the diagonal entries are zero. Hence, the graph is undirected by construction and does not contain self-loops. AMEI graphs extend the class of edge-Markovian graphs [27] and allow us to model a wider class of edge processes. For example, in an edge-Markovian graph, the time it takes for an edge to switch from connected to disconnected (or vice versa) follows an exponential distribution. In contrast, in an AMEI graph, we can design the function $g_{i j}$ and the Markov process $\theta_{i j}$ to fit any desired distribution for the contact durations with arbitrary precision, as illustrated in the following example:

Example 2.3. Let $\theta$ be the Markov process over the state space $S=\left\{c_{1}, \ldots, c_{n}, d_{1}, \ldots, d_{m}\right\}$ presenting the statetransition diagram shown in Fig. 1 Consider an edge modeled by the aggregated Markov process $\sigma=f(\theta)$, where the function $f: S \rightarrow\{0,1\}$ is defined by $f\left(c_{i}\right)=1$ and $f\left(d_{j}\right)=0$ for all $i \in[n]$ and $j \in[m]$. Therefore, the edge is active if and only if $\theta$ is in one of the states $c_{1}, \ldots, c_{n}$. From the diagram in Fig. 1, we see that the edge is active from the time $\theta$ enters $c_{1}$ until the time $\theta$ arrives to $d_{1}$. It can be proved that the time elapsed between these two events follows a Coxian probability distribution, which forms a dense subset in the set of nonnegative random variables [42]. Therefore, by appropriately choosing the transition rates $p_{1}, \ldots, p_{n-1}, q_{1}$, $\ldots, q_{n}$, it is possible to tune the distribution followed by the time the edge is active in order to follow any distribution with an arbitrary accuracy (when $n$ is large enough). From the symmetry of the diagram in Fig. 1. we can also tune the parameters $r_{1}, \ldots, r_{m-1}, s_{1}, \ldots$, $s_{m}$ so that the time the edge is inactive follows any given probability distribution with an arbitrary accuracy.

In the following definition, we introduce a directed version of AMEI graphs.

Definition 2.4. Consider a collection of aggregated Markov processes $\sigma_{i j}=g_{i j}\left(\theta_{i j}\right)$ where $g_{i j}$ are $\{0,1\}$-valued functions and $\theta_{i j}$ are stochastically independent Markov processes for $i, j \in[n]$ and $i \neq j$. An aggregatedMarkovian arc-independent (AMAI) network is a random graph process in which $A_{i j}=\sigma_{i j}$ for $i \neq j$ and $A_{i i}=0$ 
for all $i \in[n]$. If $\sigma_{i j}$ is irreducible for all possible pairs $(i, j)$, then we say that $\mathcal{G}$ is irreducible.

Once the class of aggregated-Markovian networks is introduced, we analyze spreading processes taking on these random graph processes.

\subsection{Problem Statement}

In this paper, we address the following question: Under what conditions does a spreading process taking place in a TV network with aggregated-Markovian link dynamics die out exponentially fast? In other words, we consider the problem of analyzing the stability of the upper-bounding linear model (1) when the graph is an aggregated-Markovian network. In this case, the entries of the adjacency matrix are random processes and, therefore, (1) is the following stochastic linear differential equation:

$$
\Sigma: \dot{p}(t)=(B A(t)-D) p(t),
$$

where $A(t)$ is a random matrix process. In the following section, we will derive conditions under which the diseasefree equilibrium of $\Sigma$ is stable, i.e., the infection probabilities converge to zero as $t \rightarrow \infty$ exponentially fast, in the following sense:

Definition 2.5. Consider a random graph process $\mathcal{G}$ defined by either an AMEI or AMAI graph. We say that the disease-free equilibrium of $\Sigma$ is almost surely exponentially stable if there exists $\lambda>0$ such that

$$
\operatorname{Pr}\left(\limsup _{t \rightarrow \infty} \frac{\log \|p(t)\|}{t} \leq-\lambda\right)=1,
$$

for all initial states $p(0)=p_{0}$ and $\sigma_{i j}(0)=\sigma_{i j, 0}$. The supremum of $\lambda$ satisfying the above condition is called the decay rate.

\section{Stability of Spreading Processes in Ran- dom Graph Processes}

In this section, we state the main results in this paper. In particular, we derive conditions for the spreading model $\Sigma$ to be stable when the network structure varies according to an AMEI (or AMAI) random graph process. In Subsection 3.1. we apply a stability condition [37 based on the direct application of the Itô formula for jump processes to derive stability conditions in terms of the largest eigenvalue of a matrix whose size depends exponentially on the number of edges in the graph. Due to the exponential size of this matrix, this condition is hard (if not impossible) to apply in the analysis of large-scale networks. Motivated by this limitation, we propose in Subsection 3.2 an alternative approach using tools from the theory of random matrices. In particular, we derive stability conditions in terms of the largest eigenvalue of a matrix whose size depends linearly on the number of nodes. In Subsection 3.3, we extend our results from continuous-time Markov processes to spreading processes modeled as discrete-time Markov chains.

\subsection{Stability Conditions: Exponential Matrix Size}

In this subsection, we show that the result in [37. Theorem 7.1] based on the direct application of the Itô formula for jump processes results in stability conditions that are not well-suited for large-scale graphs. We illustrate this idea through the analysis of the stability of the spread when $\mathcal{G}$ is an AMAI random graph process. For simplicity in our exposition, we here temporarily assume that all the processes $\sigma_{i j}$ are Markovian; i.e., the mappings $g_{i j}$ are the identities.

To state our claims, we need to introduce the following notations. Let $\mathcal{G}$ be an AMAI random graph process (Definition 2.4). Let $\mathcal{E}$ be the set of (time-varying) directed edges in $\mathcal{G}$ (i.e., those directed edges such that $\sigma_{i j}$ is not the zero stochastic process). Let $m=|\mathcal{E}|$ be the number of diedges in $\mathcal{G}$ and label the diedges using integers $1, \ldots, m$. Recall that these edges are not always present in the graph, but they appear (link is 'on') and disappear (link is 'off') according to a collection of aggregated-Markov processes. Therefore, at a particular time instant, only a subset of the edges in $\mathcal{E}$ are present in the graph. The set of present edges can be represented by an element of $\{0,1\}^{m}$, since we have $m$ possible edges that can be either 'on' of 'off'. In other words, at a particular time, the contact network is one of the $2^{m}$ possible subgraphs of $\mathcal{G}$.

To analyze the resulting dynamics, we consider the set of $2^{m}$ possible subgraphs of $\mathcal{G}$ and label them using integers $\left\{1, \ldots, 2^{m}\right\}$. We define the one-to-one function $\chi:\left\{1, \ldots, 2^{m}\right\} \rightarrow\{0,1\}^{m}$, as the function that maps a particular subgraph of $\mathcal{G}$ into the binary string in $\{0,1\}^{m}$ that indicates the subset of present edges in the subgraph. For the subgraph labeled $\ell$, we denote by $\chi_{\ell}$ the corresponding binary sequence and by $\chi_{\ell}(k)$ the $k$-th entry in this sequence. In other words, $\chi_{\ell}(k)=1$ means that the edge labeled $k$ in $\mathcal{G}$ is present in the subgraph labeled $\ell$. Furthermore, we denote by $F_{\ell} \in\{0,1\}^{n \times n}$ the adjacency matrix representing the structure of the subgraph labeled $\ell$.

Given two labels $\ell, \ell^{\prime} \in\left[2^{m}\right]$, define the edit distance $d\left(\ell, \ell^{\prime}\right)$ to be the minimum number of links that must be added and/or removed from the subgraph labeled $\ell$ to construct the subgraph labeled $\ell^{\prime}$, i.e., $d\left(\ell, \ell^{\prime}\right)=\left|\left\{k \in[m]: \chi_{\ell}(k) \neq \chi_{\ell^{\prime}}(k)\right\}\right|$. If $d\left(\ell, \ell^{\prime}\right)=1$ (i.e., the subgraphs differ in a single diedge), we define $k_{\ell, \ell^{\prime}}$ as the (only) index $k$ such that $\chi_{\ell}(k) \neq \chi_{\ell^{\prime}}(k)$ (i.e., the label of the single edge that must added/removed). Finally, assume that the transition probabilities of the Markov process $\sigma_{i j}$ $((i, j) \in \mathcal{E})$ are given by

$$
\begin{aligned}
& \operatorname{Pr}\left(\sigma_{i j}(t+h)=1 \mid \sigma_{i j}(t)=0\right)=u_{i j} h+o(h), \\
& \operatorname{Pr}\left(\sigma_{i j}(t+h)=0 \mid \sigma_{i j}(t)=1\right)=v_{i j} h+o(h),
\end{aligned}
$$

for some nonnegative constants $u_{i j}$ and $v_{i j}$. We define $u(k)=u_{i_{k} j_{k}}$ and $v(k)=v_{i_{k} j_{k}}$ for each $k \in[m]$.

Under the above notations, the following stability condition readily follows from [37. Theorem 7.1]:

Proposition 3.1. Let $\mathcal{G}$ be an AMAI random graph process. Define $\Pi \in \mathbb{R}^{2^{m} \times 2^{m}}$ entry-wise, as follows. For $\ell \neq \ell^{\prime}$,

$$
\Pi_{\ell \ell^{\prime}}= \begin{cases}u\left(k_{\ell, \ell^{\prime}}\right), & \text { if } d\left(\chi_{\ell}, \chi_{\ell^{\prime}}\right)=1 \text { and } \chi_{\ell}\left(k_{\ell, \ell^{\prime}}\right)=0, \\ v\left(k_{\ell, \ell^{\prime}}\right), & \text { if } d\left(\chi_{\ell}, \chi_{\ell^{\prime}}\right)=1 \text { and } \chi_{\ell}\left(k_{\ell, \ell^{\prime}}\right)=1, \\ 0, & \text { otherwise. }\end{cases}
$$


For $\ell=\ell^{\prime}$, we let $\Pi_{\ell \ell}=-\sum_{\ell^{\prime} \neq \ell} \Pi_{\ell \ell^{\prime}}$. Then, for every $i$, the probability that $i$ is infected at time $t$ in the HeNeSIS model converges to zero exponentially fast as $t \rightarrow \infty$ if the $\left(n 2^{m}\right) \times\left(n 2^{m}\right)$ matrix

$$
\Pi \otimes I_{n}+\bigoplus_{\ell=1}^{2^{m}}\left(B F_{\ell}-D\right)
$$

where $\otimes$ denotes the Kronecker product of matrices, is Hurwitz stable.

As stated before, this stability condition is not applicable to large-scale networks, since it requires the computation of the eigenvalue with the largest real part of a matrix whose size increases exponentially with the number of edges in the graph $\mathcal{G}$. In the following subsection, we present alternative stability conditions that are better-suited for stability analysis of large networks.

\subsection{Stability Analysis: Linear Matrix Size}

In this subsection, we present sufficient conditions for almost sure exponential stability of the disease-free equilibrium of $\Sigma$ when the underlying time-varying network is described by an AMEI (or AMAI) random graph process. For clarity in our exposition, we state and discuss our main results in this section, leaving the details of the proofs for Subsections 5.1 and 5.2 .

We now state our main results. Let us consider two positive constants $b$ and $d$, and define the decreasing function $\kappa_{b, d}:[0, \infty) \rightarrow(0, n]$ by

$$
\kappa_{b, d}(s)=n e^{s / b}\left(\frac{b s+d}{d}\right)^{-\frac{b s+d}{b^{2}}},
$$

where $n$ is the number of the nodes in the network. The following theorem provides conditions for a spreading process taking place in an AMAI random graph process to be almost surely exponentially stable.

Theorem 3.2. Let $\mathcal{G}$ be an irreducible AMAI random graph process and define $\bar{A} \in \mathbb{R}^{n \times n}$ component-wise, as follows

$$
\begin{aligned}
& \bar{A}_{i j}=\lim _{t \rightarrow \infty} \operatorname{Pr}\left(A_{i j}(t)=1\right) . \\
& \text { Let } \bar{\beta}=\max _{1 \leq i \leq n} \beta_{i}, \underline{\delta}=\min _{1 \leq i \leq n} \delta_{i} \text {, and } \\
& \Delta_{1}=\max _{1 \leq i \leq n} \sum_{j=1}^{n}\left(\beta_{i}^{2} \bar{A}_{i j}\left(1-\bar{A}_{i j}\right)+\beta_{j}^{2} \bar{A}_{j i}\left(1-\bar{A}_{j i}\right)\right), \\
& c_{1}=\mu(B(\operatorname{sgn} \bar{A})-D)-\frac{\kappa_{\bar{\beta}, \Delta_{1}}^{-1}(1)}{2} \text {, } \\
& \bar{s}_{1}=2 \underline{\delta}+2 c_{1}^{-} \text {. }
\end{aligned}
$$

Define

$$
\tau_{A}=\operatorname{maximize}_{s \in\left(\kappa_{\bar{\beta}, \Delta_{1}}^{-1}(1), \bar{s}_{1}\right]}\left(-\frac{s+2 c_{1} \kappa_{\bar{\beta}, \Delta_{1}}(s)}{2\left(1-\kappa_{\bar{\beta}, \Delta_{1}}(s)\right)}\right) .
$$

If

$$
\mu(B \bar{A}-D)<\tau_{A},
$$

then the disease-free equilibrium of $\Sigma$ is almost surely exponentially stable with a decay rate greater than or equal to

$$
-\mu(B \bar{A}-D)\left(1-\kappa_{\bar{\beta}, \Delta_{1}}\left(s^{*}\right)\right)-\left(s^{*} / 2\right)-c_{1} \kappa_{\bar{\beta}, \Delta_{1}}\left(s^{*}\right),
$$

where $s^{*}$ denotes the optimal value of $s$ in the maximization problem (7).

Proof: See Subsection 5.1

Remark 3.3. The existence of the limit in (6) is guaranteed by the irreducibility of $\mathcal{G}$ as will be discussed in Subsection 5.1. where we will also give an explicit representation of the limit.

The major computational cost for checking the stability condition in (8) comes from that of finding the matrix measure $\mu(B \bar{A}-D)$. Since the matrix $B \bar{A}-D$ has size $n$, we can compute $\mu(B \bar{A}-D)$ in $O\left(n m^{2}\right)$ operations using Lanczos's algorithm [43], where $n$ and $m$ are the number of nodes and diedges in $\mathcal{G}$. In contrast, computing the dominant eigenvalue of the $n 2^{m}$-dimensional matrix in (4) requires $O\left(n m^{2} 2^{m}\right)$ operations, since this matrix has $O\left(m 2^{m}\right)$ nonzero entries. Therefore, the result in Theorem 3.2 (based on random graph-theoretical results) represents a major computational improvement with respect to the result in Proposition 3.1 (based on a direct application of the Itô formula for jump processes [37|).

Above, we have analyzed the stability of spreading processes in aggregated-Markovian arc-independent (AMAI) networks. In the rest of the subsection, we derive stability conditions for spreading processes taking place in aggregated-Markovian edge-independent (AMEI) networks. The next theorem is the AMEI counterpart of Theorem 3.2

Theorem 3.4. Let $\mathcal{G}$ be an irreducible AMEI random graph process. Let

$$
\begin{aligned}
\Delta_{2} & =\max _{1 \leq i \leq n} \sum_{j=1}^{n}\left(\beta_{i} \beta_{j} \bar{A}_{i j}\left(1-\bar{A}_{i j}\right)\right), \\
c_{2} & =\eta(B(\operatorname{sgn} \bar{A})-D)-\kappa_{\bar{\beta}, \Delta_{2}}^{-1}(1), \\
\bar{s}_{2} & =\underline{\delta}+c_{2}^{-}
\end{aligned}
$$

Define

$$
\tau_{E}=\operatorname{maximize}_{s \in\left(\kappa_{\bar{\beta}, \Delta_{2}}^{-1}(1), \bar{s}_{2}\right]}\left(-\frac{s+c_{2} \kappa_{\bar{\beta}, \Delta_{2}}(s)}{1-\kappa_{\bar{\beta}, \Delta_{2}}(s)}\right) .
$$

If

$$
\eta(B \bar{A}-D)<\tau_{E},
$$

then the disease-free equilibrium of $\Sigma$ is almost surely exponentially stable with a decay rate greater than or equal to

$$
-\eta(B \bar{A}-D)\left(1-\kappa_{\bar{\beta}, \Delta_{2}}\left(s^{*}\right)\right)-s^{*}-c_{2} \kappa_{\bar{\beta}, \Delta_{2}}\left(s^{*}\right),
$$

where $s^{*}$ denotes the optimal value of $s$ in the maximization problem (10).

Proof: See Subsection 5.2

Theorems 3.2 and 3.4 provide threshold conditions for stability of spreading processes in time-varying networks when agents present heterogeneous infection and recovery rates, $\beta_{i}$ and $\delta_{i}$, respectively. In the particular case of networks with homogeneous rates, i.e., $\beta_{i}=\beta$ and $\delta_{i}=\delta$ for all $i$, our results have an appealing interpretation that can be related with existing results in the literature. The following theorem states a threshold stability condition in the homogeneous case: 
Theorem 3.5. Let $\mathcal{G}$ be an irreducible AMEI random graph process. Assume that $\beta_{i}=\beta>0$ and $\delta_{i}=\delta>0$ for all $i \in[n]$. Let

$$
\begin{aligned}
\Delta_{3} & =\max _{1 \leq i \leq n} \sum_{j=1}^{n}\left(\bar{A}_{i j}\left(1-\bar{A}_{i j}\right)\right), \\
c_{3} & =\eta(\operatorname{sgn} \bar{A})-\kappa_{1, \Delta_{3}}^{-1}(1), \\
\bar{s}_{3} & =\frac{\delta}{\beta}+c_{3}^{-} .
\end{aligned}
$$

Define

$$
\xi_{H}=\operatorname{maximize}_{s \in\left(\kappa_{1, \Delta_{3}}^{-1}(1), \bar{s}_{3}\right]} \frac{1-(\beta / \delta)\left(s+c_{3} \kappa_{1, \Delta_{3}}(s)\right)}{1-\kappa_{1, \Delta_{3}}(s)} .
$$

If

$$
\frac{\beta}{\delta}<\frac{\xi_{H}}{\eta(\bar{A})},
$$

then the disease-free equilibrium of $\Sigma$ is almost surely exponentially stable with decay rate greater than or equal to

$$
(\delta / \beta)-\eta(\bar{A})-s^{*}-\left(c_{3}-\lambda_{3}\right) \kappa_{1, \Delta_{3}}\left(s^{*}\right),
$$

where $s^{*}$ denotes the optimal value of $s$ in the optimization problem (14). Moreover, if

$$
\frac{\beta}{\delta} \geq \frac{1}{\eta(\operatorname{sgn} \bar{A})},
$$

then $\xi_{H}<1$.

\section{Proof: See Subsection 5.2}

Remark 3.6. Since $A(t) \leq \operatorname{sgn} \bar{A}$ entry-wise for every $t \geq 0$, the solution of $\Sigma$ is always upper-bounded by that of the deterministic differential equation $\dot{p}(t)=(\beta \operatorname{sgn} \bar{A}-\delta I) p(t)$, whose solution converges to zero as $t \rightarrow \infty$ if and only if $\beta / \delta<1 / \eta(\operatorname{sgn} \bar{A})$. Therefore, if the inequality 16 is false, then the disease-free equilibrium of $\Sigma$ is almost surely exponentially stable and, hence, we do not need to check condition (15). In other words, condition (16) guarantees that stability analysis of $\Sigma$ is not a trivial problem.

As shown in Subsection 2.2, the epidemic threshold in a static network of agents with homogeneous rates is given by $\beta / \delta<1 / \eta(A)$. Condition (15) provides a similar epidemic threshold for time-varying networks in terms of the spectral abscissa of $\bar{A}$, which can be interpreted as an aggregated static network based on long-time averages. Furthermore, $\xi_{H}$ is a multiplicative factor that modifies the epidemic threshold corresponding to the aggregated static network. Below, we derive explicit expressions of $\eta(\bar{A})$ for a few particular examples of time-varying graph models found in the literature:

Example 3.7 (Dynamic small-world networks). We consider a time-varying version of the small-world network model studied in [44]. The network consists of $n$ nodes and $n$ static edges in the set $\mathcal{E}_{0}=\{(1,2),(2,3), \ldots,(n-$ $1, n),(n, 1)\}$ (see Fig. 2). Apart from these static edges, we also consider a collection of dynamic edges that switch on and off over time. In particular, we allow any other pair of nodes $(i, j) \notin \mathcal{E}_{0}(i \neq j)$ to be

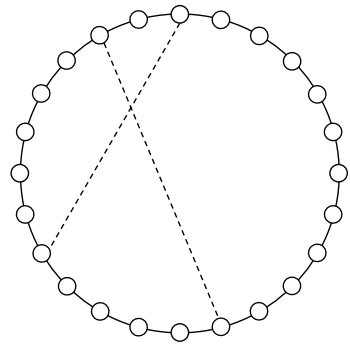

Fig. 2. Dynamic small-world network. Solid lines represent static edges, while dashed lines represent temporarily active edges modeled by aggregated-Markov processes.

dynamically connected according to an irreducible aggregated Markov process $a_{i j}=g_{i j}\left(\theta_{i j}\right)$. For simplicity in our analysis, we assume that the stationary probability of an edge $(i, j) \notin \mathcal{E}_{0}$ being active, defined by $r_{i j}=\lim _{t \rightarrow \infty} \operatorname{Pr}\left(a_{i j}(t)=1\right)$, equals a constant $r>0$ independent of $i$ and $j$. In this case, the matrix $\bar{A}$ takes the form

$$
\bar{A}_{i j}= \begin{cases}0, & \text { if } i=j, \\ 1, & \text { if }(i, j) \in \mathcal{E}_{0} \\ r, & \text { otherwise. }\end{cases}
$$

The spectral abscissa of $\bar{A}$ is given by $1+r(n-2)$. Therefore, the stability condition (15) reads $\beta / \delta<\xi_{H} /(1+$ $r(n-2))$.

Example 3.8 (Edge-Markovian graph). In this example, we consider the edge-Markovian graph model proposed in [27]. In this model, all the undirected edges $(i, j)$ $(i<j)$ are time-varying and modeled by independent $\{0,1\}$-valued Markov processes $\theta_{i j}$ sharing the same activation rate $q>0$ and de-activation rate $r>0$. In this case, we have

$$
\bar{A}_{i j}= \begin{cases}0, & \text { if } i=j, \\ q /(q+r), & \text { otherwise, }\end{cases}
$$

and therefore $\eta(\bar{A})=(n-1) q /(q+r)$. The stability condition (15) hence reads $\beta / \delta<\xi_{H}(q+r) /((n-1) q)$.

In what follows, we elaborate on the behavior of the factor $\xi_{H}$. In particular, we will analyze its dependence on $\Delta_{3}$ defined in (13). Notice that $\Delta_{3}$ is zero when $\bar{A}_{i j}$ is either 0 or 1 for all pairs $(i, j)$, which corresponds to the case of a static, deterministic graph $\mathcal{G}$. In contrast, the maximum value of $\Delta_{3}$ is achieved when $\bar{A}_{i j}=1 / 2$, i.e., edges are either 'on' or 'off' with equal probability (asymptotically). Therefore, the term $\Delta_{3}$ can be interpreted as a measure of structural variability, in the long run. In Fig. 3, we illustrate the behavior of the factor $\xi_{H}$ as we modify the network size $n$, the epidemic ratio $\delta / \beta$, and the uncertainty measure $\Delta_{3}$. We observe that the smaller the value of $\Delta_{3}$, the larger the value of $\xi_{H}$. In other words, as we reduce the structural variability (i.e., we reduce $\Delta_{3}$ ), we obtain a better approximation using an aggregated static model (i.e., $\xi_{H}$ approaches 1). Furthermore, notice that the value of $\xi_{H}$ tends to 1 as $n$ increases. This indicates that the aggregated static network approximates the epidemic threshold more accurately as the network grows. 


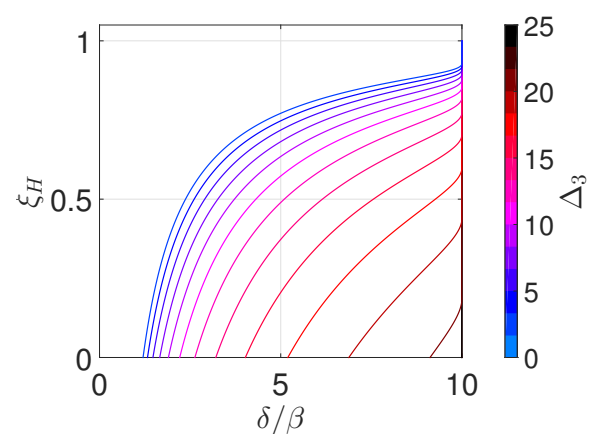

(a)

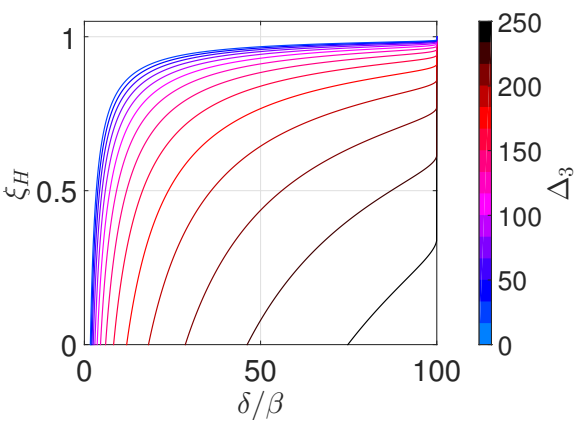

(b)

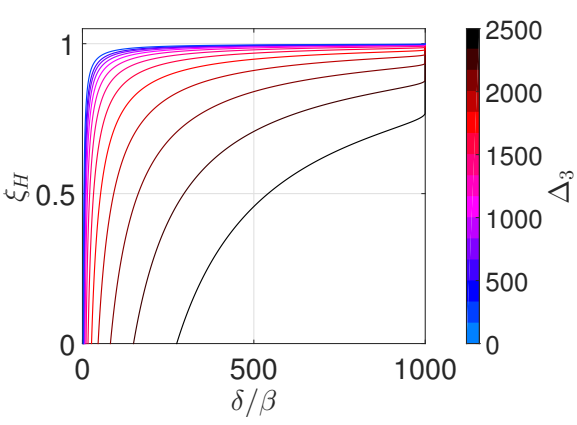

(c)

Fig. 3. Plots of $\xi_{H}$ as $\delta / \beta$ and $\Delta_{3}$ vary for the following cases: (a) $n=100$ and $\eta(\operatorname{sgn} \bar{A})=10$, (b) $n=1,000$ and $\eta(\operatorname{sgn} \bar{A})=100$, and (c) $n=10,000$ and $\eta(\operatorname{sgn} \bar{A})=1,000$.

In this section, we have introduced our main theoretical results, namely, we have studied the epidemic threshold in the wide class of aggregated-Markovian random graph processes. Before we illustrate our results with some numerical simulations in Section 4 , we will discuss the case when the epidemics is modeled as a discrete-time stochastic process.

\subsection{Discrete-Time Epidemic Dynamics}

In this subsection, we briefly present a discrete-time version of the stability analysis provided above. We define a discrete-time dynamic random graph as a stochastic process $\mathcal{G}=\{\mathcal{G}(k)\}_{k=0}^{\infty}$ taking values in the set of directed graphs with $n$ nodes. In the discrete-time case, aggregatedMarkovian edge- and arc-independent dynamic random graphs are defined in the same way as in the continuoustime case. Let us consider the discrete-time epidemic model in (3) taking place in the dynamics random graph $\mathcal{G}$. The resulting dynamics is described in the following stochastic difference equation:

$$
\Sigma_{d}: p(k+1)=(B A(k)+I-D) p(k),
$$

where $A(k)$ denotes the adjacency matrix of $\mathcal{G}(k)$.

We define almost sure exponential stability of the disease-free equilibrium of $\Sigma_{d}$ in the same way as we did for the continuous-time case:

Definition 3.9. Let $\mathcal{G}$ be a discrete-time edge-independent dynamic random graph. We say that the disease-free equilibrium of $\Sigma_{d}$ is almost surely exponentially stable if there exists $\lambda>0$ such that

$$
\operatorname{Pr}\left(\limsup _{k \rightarrow \infty} \frac{\log \|p(k)\|}{k} \leq-\lambda\right)=1
$$

for all $p(0)=p_{0} \in[0,1]^{n}$ and $\sigma_{i j}(0)=\sigma_{i j, 0}(1 \leq i<$ $j \leq n$ ). We call the supremum of $\lambda$ satisfying the above condition the decay rate.

For simplicity in our exposition, we focus only on the edge-independent case (AMEI graph process), since the analysis is identical for the arc-independent case. The next theorem provides a sufficient condition for almost sure exponential stability of the disease-free equilibrium of $\Sigma_{d}$.
Theorem 3.10. Consider an irreducible and aperiodif 2 AMEI random graph process $\mathcal{G}$ in discrete-time. Let $\lambda_{4}=$ $\eta(B \bar{A}+I-D)$ and $M_{\max }=B(\operatorname{sgn} \bar{A})+I-D$. Define

$$
\tau_{D}=\underset{0 \leq s \leq 1-\lambda_{4}}{\operatorname{maximize}}\left(\left(\frac{\lambda_{4}}{\eta\left(M_{\max }\right)}\right)^{\kappa_{\bar{\beta}, \Delta_{2}}(s)}-s\right),
$$

where $\Delta_{2}$ is defined in 9 . If

$$
\lambda_{4}<\tau_{D},
$$

then the disease-free equilibrium of $\Sigma_{d}$ is almost surely exponentially stable with decay rate greater than or equal to

$$
\gamma_{D}=-\log \left(\lambda_{4}+s^{*}\right)-\kappa_{\bar{\beta}, \Delta_{2}}\left(s^{*}\right) \log \frac{\eta\left(M_{\max }\right)}{\lambda_{4}},
$$

where $s^{*}$ denotes the value of $s$ giving the solution to the optimization problem (17).

Proof: See Subsection 5.3

\section{Numerical Simulations}

In this section, we illustrate the effectiveness of our results with numerical simulations. In our illustrations, we consider a discrete-time edge-independent dynamic random graph $\mathcal{G}$ with $n$ nodes. This random graph process is specified by the two following rules:

1) Construct an undirected Erdős-Rényi random graph over the nodes of $\mathcal{G}$ [45]. Denote the resulting $n \times n$ adjacency matrix by $A$.

2) If $A_{i j}=1$, then nodes $i$ and $j$ are connected in $\mathcal{G}$ via a stochastic link described by a $\{0,1\}$-valued time-homogeneous Markov chain $\sigma_{i j}$. The transition probability from 0 to 1 in this Markov process is given by $q_{i j} \geq 0$; while the transition probability from 1 to 0 is given by $r_{i j} \geq 0$. If $A_{i j}=0$, then nodes $i$ and $j$ are not connected in $\mathcal{G}$ at any time.

One can easily deduce that, according to the second rule, $\bar{A}_{i j}=\bar{A}_{j i}=q_{i j} /\left(r_{i j}+q_{i j}\right)$ if $A_{i j}=1$. In the following simulations, we let $n=500$ and consider a realization of an

2. We say that an AMEI random graph process in discrete-time is aperiodic if all the aggregated Markov processes in the adjacency matrix of the graph are the images of aperiodic Markov chains. 


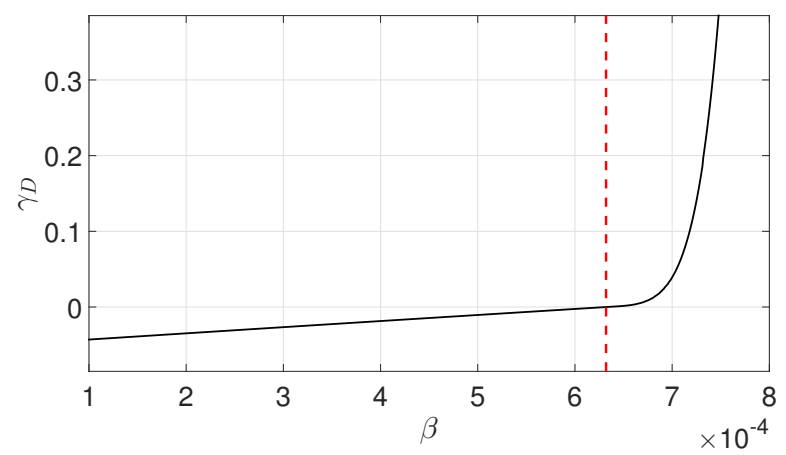

Fig. 4. The upper bound $\gamma_{D}$ of decay rates. Red dashed line shows the epidemic threshold given by Theorem 3.10

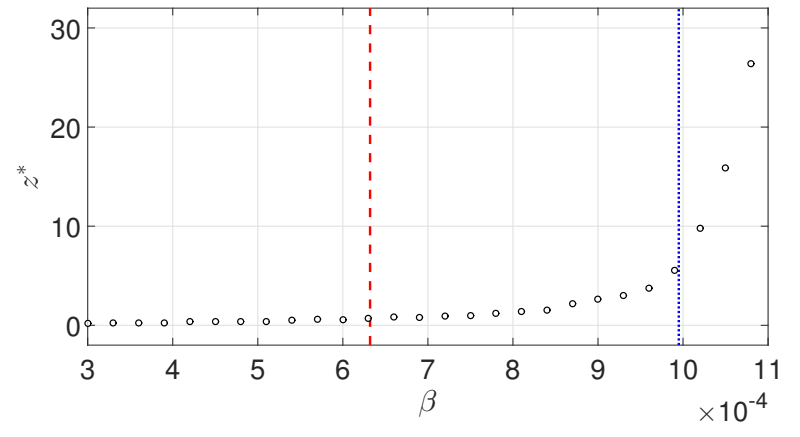

Fig. 5. The averaged numbers of the infected nodes at time $k=1000$ versus $\beta$. Red dashed line: Epidemic threshold predicted by Theorem 3.10 Blue dotted line: Epidemic threshold predicted by using aggregated static network $\bar{A}$.

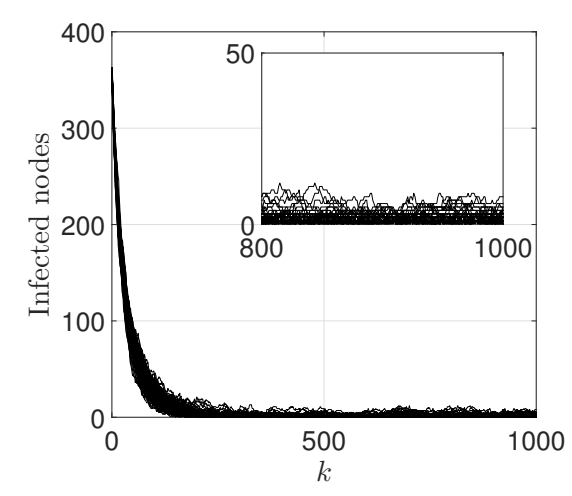

(a) $\beta=6.0 \times 10^{-4}$

Fig. 6. Sample paths of the number of infected nodes for different values of $\beta$.

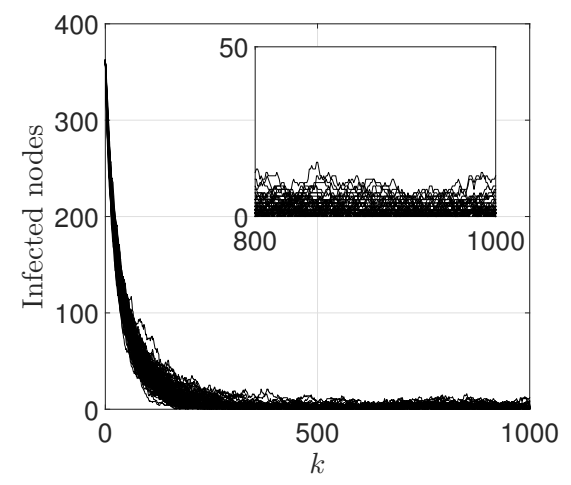

(b) $\beta=7.5 \times 10^{-4}$

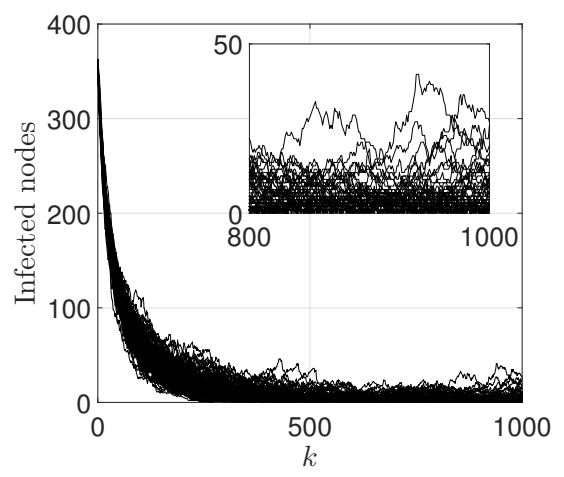

(c) $\beta=9.0 \times 10^{-4}$ the Erdős-Rényi graph with an edge probability of 0.2 (in step 1 above). The values of the transition probabilities $r_{i j}$ (in step 2 above) are heterogeneous over the links of the Erdős-Rényi graph. In particular, we have chosen for $r_{i j}$ a collection of random values from the Gaussian $\mathcal{N}(1 / 2,1 / 8)$, where we have truncated those values $r_{i j}>1$ to $r_{i j}=1$, and $r_{i j}<0$ to $r_{i j}=0$. Finally, we let $q_{i j}=1-r_{i j}$.

We consider a discrete-time spreading process with homogeneous infection and recovery rates, i.e., $\beta_{i}=\beta$ and $\delta_{i}=0.05$ for all $i$. Using Theorem 3.10, we deduce that the disease-free equilibrium of $\Sigma_{d}$ is almost surely exponentially stable if $\beta<6.32 \times 10^{-4}$. The upper bound $\gamma_{D}$ of the decay rate versus $\beta$ is shown in Fig. 4 . In order to check the accuracy of this epidemic threshold, we compare this threshold with the one obtained from the following simulation. We simulate the spreading process over the dynamic random graph described above for various values of $\beta$. For each value of $\beta$, we generate 500 sample paths of the spreading process. In this simulation, we prevent a spreading process from dying out by re-infecting a randomly chosen node immediately after the infection process dies (i.e., when all the nodes become susceptible). A similar reinfection mechanism is employed for simulating spreading processes over static networks [46]. After the simulation, we compute the averaged number of infected nodes $y^{*}$ at time $k=1,000$. This is the meta-stable number of infected nodes when re-infection of nodes is allowed. We then determine the metastable number of infected nodes in the original spreading process without reinfection as $z^{*}=y^{*}-1$, where the subtraction of one compensates the effect of re-infection. Finally, we define the empirical epidemic threshold $\beta^{*}$ as the maximum value of $\beta$ such that $z^{*}<1$.

Fig. 5 shows the value of $z^{*}$ as $\beta$ varies. We see that the above defined empirical threshold $\beta^{*}$ lies between $7.2 \times 10^{-4}$ and $7.5 \times 10^{-4}$, confirming that our condition indeed gives a sufficient condition for stability. On the other hand, the epidemic threshold based on the aggregated static network $\bar{A}$ (i.e., the supremum of $\beta$ such that $\beta / \delta<1 / \eta(\bar{A})$ ) is $9.95 \times 10^{-4}$ and, therefore, overestimates the actual epidemic threshold. In Fig. 6, we show multiple realizations of the sample paths used to obtain Fig. 5 for $\beta=6.0 \times 10^{-4}$, $7.5 \times 10^{-4}$, and $9.0 \times 10^{-4}$.

\section{Proofs of Main Results}

This section presents the proofs of the theorems presented in Section 3

\subsection{Proof of Theorem 3.2}

We begin by recalling a basic result on the stability analysis of a class of stochastic differential equations called switched linear systems. Let $\sigma=f(\theta)$ be an aggregated Markov process defined by the mapping $f: \Lambda \rightarrow \Gamma$ and a Markov process $\theta$ with state space $\Lambda$. For each $\gamma \in \Gamma$, there is an associated state matrix $A_{\gamma} \in \mathbb{R}^{n \times n}$. An aggregated Markov 
jump linear system is defined by the following stochastic differential equation

$$
\dot{x}(t)=A_{f(\theta(t))} x(t),
$$

where $x(0)=x_{0} \in \mathbb{R}^{n}$ and $\theta(0)=\theta_{0} \in \Lambda$. We remark that, if $f$ is the identity mapping, then the system in (20) is a Markov jump linear system [47]. We say that the system in (20) is almost surely exponentially stable if there exists $\lambda>0$ such that $\operatorname{Pr}\left(\lim \sup _{t \rightarrow \infty} t^{-1} \log \|x(t)\| \leq-\lambda\right)=1$ for all $x_{0}$ and $\theta_{0}$. The supremum of $\lambda$ satisfying the above condition is called the decay rate. In order to prove Theorem 3.2. we will need the following criterion for almost sure exponential stability of aggregated Markov jump linear systems.

Lemma 5.1. Assume that $\theta$ is an irreducible Markov process and let $\pi$ be its unique stationary distribution. Assume that

$$
E\left[\mu\left(A_{f(\pi)}\right)\right]<0 .
$$

Then, the aggregated Markov jump linear system in 20 is almost surely exponentially stable with a decay rate greater than or equal to $-E\left[\mu\left(A_{f(\pi)}\right)\right]$.

Proof: The above statement is known to be true for Markov jump linear systems [48. Theorem 4.2], i.e., when $f$ is the identity mapping. Let us now consider an arbitrary function $f$. Define $B_{\lambda}=A_{f(\lambda)}$ for each $\lambda \in \Lambda$. Then, we see that the system in 20] is equivalent to the Markov jump linear system $\dot{x}(t)=B_{\theta(t)} x(t)$, for which we can guarantee almost sure exponential stability with decay rate grater than or equal to $-E\left[\mu\left(B_{\pi}\right)\right]>0$ via the condition $E\left[\mu\left(B_{\pi}\right)\right]<0$ [48. Theorem 4.2]. This condition is equivalent to (21) by the definition of $B_{\lambda}$.

Using Lemma 5.1, we can prove the following preliminary result, which will be useful in the proof of Theorem 3.2

Proposition 5.2. Consider an irreducible AMAI random graph process $\mathcal{G}$. For all distinct and ordered pairs $(i, j) \in[n]^{2}$, let $h_{i j}$ be an independent Bernoulli random variable with mean $\bar{A}_{i j}$. Define the random matrix

$$
M_{1}=-D+\sum_{i=1}^{n} \sum_{j \neq i} \beta_{i} h_{i j} E_{i j},
$$

where $E_{i j}$ denotes the $\{0,1\}$-matrix whose entries are all zero except its $(i, j)$-entry. If $E\left[\mu\left(M_{1}\right)\right]<0$, then, the infection-free equilibrium of $\Sigma$ is almost surely exponentially stable with decay rate greater than or equal to $-E\left[\mu\left(M_{1}\right)\right]$.

Proof: Define the direct product $\theta=\bigotimes_{i=1}^{n} \bigotimes_{j \neq i} \theta_{i j}$, which is a stochastic process with state space $\Lambda=$ $\bigotimes_{i=1}^{n} \bigotimes_{j \neq i} \Lambda_{i j}$. Define $f: \Lambda \rightarrow\{0,1\}^{n(n-1)}$ by $(f(\lambda))_{i j}=$ $f_{i j}\left(\lambda_{i j}\right)$. Also, for $\gamma=\left(\gamma_{i j}\right)_{i, j} \in\{0,1\}^{n(n-1)}$, define the matrix

$$
F_{\gamma}=\sum_{i=1}^{n} \sum_{j \neq i} \gamma_{i j} E_{i j}
$$

Then, from Definition 2.4. we see that $A(t)=$ $\sum_{i=1}^{n} \sum_{j \neq i} \sigma_{i j}(t) E_{i j}=F_{\sigma(t)}=F_{f(\theta(t))}$. Therefore, we can rewrite $\Sigma$ as the aggregated Markov jump linear system $\Sigma_{1}: \dot{p}(t)=\left(B F_{f(\theta(t))}-D\right) p(t)$.

By the irreducibility of $\mathcal{G}$, each time-homogeneous Markov process $\theta_{i j}$ has a unique stationary distribution $\pi_{i j}$ on $\Lambda_{i j}$. Then, the unique stationary distribution of $\theta$ is $\pi=\bigotimes_{i=1}^{n} \bigotimes_{j \neq i} \pi_{i j}$. Therefore, by Lemma 5.1. if $E\left[\mu\left(B F_{f(\pi)}-D\right)\right]<0$, then $\Sigma_{1}=\Sigma$ is almost surely exponentially stable with decay rate greater than or equal to $-E\left[\mu\left(B F_{f(\pi)}-D\right)\right]$. Therefore, to complete the proof of the proposition, we need to show that

$$
B F_{f(\pi)}-D=M_{1},
$$

in the sense that the random matrices appearing in the both sides of the equation share the same probability distribution. From [23] and the definition of the matrix $B$, we have

$$
B F_{f(\pi)}-D=-D+\sum_{i=1}^{n} \sum_{j \neq i} \beta_{i} f_{i j}\left(\pi_{i j}\right) E_{i j}
$$

Since $f_{i j}$ maps into $\{0,1\}$, the random variable $f_{i j}\left(\pi_{i j}\right)$ is the Bernoulli random variable with mean equal to

$$
\begin{aligned}
\operatorname{Pr}\left(f_{i j}\left(\pi_{i j}\right)=1\right) & =\pi_{i j}\left(f_{i j}^{-1}(\{1\})\right) \\
& =\lim _{t \rightarrow \infty} \operatorname{Pr}\left(\theta_{i j}(t) \in f_{i j}^{-1}(\{1\})\right) \\
& =\lim _{t \rightarrow \infty} \operatorname{Pr}\left(\sigma_{i j}(t)=1\right) \\
& =\bar{A}_{i j},
\end{aligned}
$$

where we used Lemma 2.1. Moreover, all the random variables $f_{i j}\left(\pi_{i j}\right)(i, j \in[n], i \neq j)$ are independent of each other because $\mathcal{G}$ is arc-independent. From these observations and the equation (25), we obtain (24), as desired.

In order to complete the proof of Theorem 3.2, we need the following result from the theory of random graphs concerning the maximum eigenvalue of the sum of random symmetric matrices.

Proposition 5.3 ([49]). Let $X_{1}, \ldots, X_{N}$ be independent random $n \times n$ symmetric matrices. Let $C$ be a nonnegative constant such that $\left\|X_{k}-E\left[X_{k}\right]\right\| \leq C$ for every $k \in[N]$ with probability one. Also let $v^{2}=\left\|\sum_{k=1}^{N} \operatorname{Var}\left(X_{k}\right)\right\|$. Then the sum $X=\sum_{k=1}^{N} X_{k}$ satisfies

$$
\operatorname{Pr}(\eta(X)>\eta(E[X])+s) \leq \kappa_{C, v^{2}}(s)
$$

for every $s \geq 0$ (where $\kappa_{b, d}(s)$ was defined in (5)).

Proof: Let $s \geq 0$ be arbitrary. Under the assumptions stated in the proposition, it is shown in [49| that, for every $\theta>0$,

$$
\operatorname{Pr}(\eta(X)>\eta(E[X])+s) \leq n \exp \left(-\theta s+\frac{1}{2} g(C \theta) \theta^{2} v^{2}\right),
$$

where $g$ is defined by $g(x)=2 x^{-2}\left(e^{x}-x-1\right)$ for $x>0$. It is easy to show that the right hand side of the inequality takes its minimum value with respect to $\theta$ when $\theta=(1 / C) \log \left(1+\left(C s / v^{2}\right)\right)$. Substituting this particular value of $\theta$ into the inequality, we readily obtain 26 .

In order to complete the proof of Theorem 3.2. we also need the next lemma concerning Metzler matrices.

Lemma 5.4 ([50, Lemma 2]). Let $A$ and $B$ be Metzler matrices. If $A \leq B$, then we have $\eta(A) \leq \eta(B)$ and $\mu(A) \leq \mu(B)$.

We can now provide a proof of Theorem 3.2

Proof of Theorem 3.2 Let $\lambda_{1}=\mu(B \bar{A}-D)$ and define $X=M_{1}+M_{1}^{\top}-2 \lambda_{1} I-\kappa_{\bar{\beta}, \Delta_{1}}^{-1}(1) I$. Notice that

$$
\eta(E[X])=-\kappa_{\bar{\beta}, \Delta_{1}}^{-1}(1)
$$


because $\eta\left(E\left[M_{1}+M_{1}^{\top}\right]\right)=2 \mu\left(E\left[M_{1}\right]\right)=2 \mu(B \bar{A}-D)=$ $2 \lambda_{1}$ by [24. Also, from the definition of $M_{1}$ in [22), we have

$$
X=\left(-2 \lambda_{1} I-2 D-\kappa_{\bar{\beta}, \Delta_{1}}^{-1}(1) I\right)+\sum_{i=1}^{n} \sum_{j \neq i} X_{i j},
$$

where $X_{i j}=\beta_{i}\left(E_{i j}+E_{j i}\right) h_{i j}(i, j \in[n]$ and $i \neq j)$ are random, independent symmetric matrices. We then apply Proposition 5.3 to the random matrix $X$, for which we choose $C=\beta$. Clearly, the first constant term of $X$ has zero variance, while a simple computation shows that $\operatorname{Var}\left(X_{i j}\right)=\beta_{i}^{2} \bar{A}_{i j}\left(1-\bar{A}_{i j}\right)\left(E_{i i}+E_{j j}\right)$. Therefore

$$
\begin{aligned}
v^{2} & =\left\|\sum_{i=1}^{n} \sum_{j \neq i} \beta_{i}^{2} \bar{A}_{i j}\left(1-\bar{A}_{i j}\right)\left(E_{i i}+E_{j j}\right)\right\| \\
& =\left\|\bigoplus_{i=1}^{n}\left(\sum_{j=1}^{n} \beta_{i}^{2} \bar{A}_{i j}\left(1-\bar{A}_{i j}\right)+\beta_{j}^{2} \bar{A}_{j i}\left(1-\bar{A}_{j i}\right)\right)\right\| \\
& =\Delta_{1} .
\end{aligned}
$$

Combining (26), (27), and (28), we obtain the estimate

$$
\operatorname{Pr}\left(\eta(X)>-\kappa_{\bar{\beta}, \Delta_{1}}^{-1}(1)+s\right) \leq \kappa_{\bar{\beta}, \Delta_{1}}(s)
$$

for $s>\kappa_{\bar{\beta}, \Delta_{1}}^{-1}(1)$.

Let $(\Omega, \mathcal{F}, \operatorname{Pr})$ be the fundamental probability space and consider the set $\Omega_{s}=\left\{\omega \in \Omega: \eta(X)>-\kappa_{\bar{\beta}, \Delta_{1}}^{-1}(1)+s\right\}$ for $s>\kappa_{\bar{\beta}, \Delta_{1}}^{-1}(1)$. If $\omega \notin \Omega_{s}$, then $\eta(X) \leq-\kappa_{\bar{\beta}, \Delta_{1}}^{-1}(1)+s$ vacuously. On the other hand, if $\omega \in \Omega_{s}$, then Lemma 5.4 gives the obvious estimate

$$
\begin{aligned}
\eta(X) & \leq 2 \mu(B(\operatorname{sgn} \bar{A})-D)-2 \lambda_{1}-\kappa_{\bar{\beta}, \Delta_{1}}^{-1}(1) \\
& =2 c_{1}-2 \lambda_{1}
\end{aligned}
$$

because $M_{1} \leq-D+\sum_{i=1}^{n} \sum_{j \neq i} \beta_{i} E_{i j}=B(\operatorname{sgn} \bar{A})-D$. Therefore, from (29) it follows that

$$
\begin{aligned}
& E[\eta(X)] \\
\leq & \left(-\kappa_{\bar{\beta}, \Delta_{1}}^{-1}(1)+s\right) \operatorname{Pr}\left(\Omega \backslash \Omega_{s}\right)+\left(2 c_{1}-2 \lambda_{1}\right) \operatorname{Pr}\left(\Omega_{s}\right) \\
\leq & -\kappa_{\bar{\beta}, \Delta_{1}}^{-1}(1)+s+\left(2 c_{1}-2 \lambda_{1}\right) \kappa_{\bar{\beta}, \Delta_{1}}(s) .
\end{aligned}
$$

By the definition of the random matrix $X$, this inequality implies that

$$
2 E\left[\mu\left(M_{1}\right)\right] \leq 2 \lambda_{1}\left(1-\kappa_{\bar{\beta}, \Delta_{1}}(s)\right)+s+2 c_{1} \kappa_{\bar{\beta}, \Delta_{1}}(s) .
$$

Now we assume that condition (8) in the theorem holds. Then, there exists $s^{*}>\kappa_{\bar{\beta}, \Delta_{1}}(1)$ such that $2\left(1-\kappa_{\bar{\beta}, \Delta_{1}}\left(s^{*}\right)\right) \lambda_{1}<-s^{*}-2 c_{1} \kappa_{\bar{\beta}, \Delta_{1}}\left(s^{*}\right)$. Therefore, when $s=s^{*}$, the inequality (31) yields that $E\left[\mu\left(M_{1}\right)\right] \leq \lambda_{1}(1-$ $\left.\kappa_{\bar{\beta}, \Delta_{1}}\left(s^{*}\right)\right)+\left(s^{*} / 2\right)+c_{1} \kappa_{\bar{\beta}, \Delta_{1}}\left(s^{*}\right)<0$ and hence we can complete the proof of Theorem 3.2 by Proposition 5.2

Remark 5.5. The upper bound $\bar{s}_{1}$ of the interval in the maximization problem (7) does not play any role in the proof of Theorem 3.2 In fact, the theorem holds true even if we replace the interval with the infinite interval $\left(\kappa_{\bar{\beta}, \Delta_{1}}^{-1}(1), \infty\right)$. The reason why we introduce the upper bound is that 1) it makes it easy for us to find the maximum and 2) it does not introduce conservativeness. To prove the second statement, it is sufficient to show that the objective function in (7) is less than $\mu(B \bar{A}-D)$ if $s>\kappa_{\bar{\beta}, \Delta_{1}}^{-1}(1)$ and $s>\bar{s}_{1}$. From the former inequality, we obtain $0<\kappa(s)<1$. Also, notice the that from Lemma 5.4 there follows the inequality $-\underline{\delta}=\mu(-D) \leq \mu(B \bar{A}-D)$. Therefore, we can estimate the objective function as

$$
\begin{aligned}
-\frac{s+2 c_{1} \kappa_{\bar{\beta}, \Delta_{1}}(s)}{2\left(1-\kappa_{\bar{\beta}, \Delta_{1}}(s)\right)}<-\frac{s}{2}-c_{1} \kappa_{\bar{\beta}, \Delta_{1}}(s) \leq \\
-\frac{\bar{s}_{1}}{2}+c_{1}^{-}=-\underline{\delta} \leq \mu(B \bar{A}-D),
\end{aligned}
$$

as desired. We can justify the upper bounds $\bar{s}_{2}$ and $\bar{s}_{3}$ in the maximization problems (10) and (14) in the same way.

Remark 5.6. It can be easily verified that the proof of Theorem 3.2 still holds true even if we define $c_{1}$ to be $\mu(B(\operatorname{sgn} \bar{A})-D)$. However, the inclusion of the term $-\kappa_{\bar{\beta}, \Delta_{1}}^{-1}(1) / 2$ into $c_{1}$, as was done in the theorem, makes the maximum in condition (8) larger, and hence can reduce the conservativeness of this condition.

\subsection{Proof of Theorems 3.4 and 3.5}

In this subsection, we prove Theorems 3.4 and 3.5 for the edge-independent case in this subsection. We begin with the following analogue of Proposition 5.2

Proposition 5.7. Consider an irreducible AMEI random graph process $\mathcal{G}$. Let $h_{i j}(1 \leq i<j \leq n)$ be independent Bernoulli random variables with mean $\bar{A}_{i j}$. Define the random matrix

$$
M_{2}=-D+\sum_{i=1}^{n} \sum_{j>i} \sqrt{\beta_{i} \beta_{j}}\left(E_{i j}+E_{j i}\right) h_{i j} .
$$

If

$$
E\left[\eta\left(M_{2}\right)\right]<0,
$$

then, the disease-free equilibrium of $\Sigma$ is almost surely exponentially stable with decay rate greater than or equal to $-E\left[\eta\left(M_{2}\right)\right]$.

Proof: Define the direct product $\theta=\bigotimes_{i=1}^{n} \bigotimes_{j>i} \theta_{i j}$, which is a Markov process having the state space $\Lambda=$ $\bigotimes_{i=1}^{n} \bigotimes_{j>i} \Lambda_{i j}$. Also, define $g: \Lambda \rightarrow\{0,1\}^{n(n-1) / 2}$ by $(g(\lambda))_{i j}=g_{i j}\left(\lambda_{i j}\right)$. For $\gamma=\left(\gamma_{i j}\right)_{i, j} \in\{0,1\}^{n(n-1) / 2}$, define the matrix

$$
G_{\gamma}=\sum_{i=1}^{n} \sum_{j>i} \gamma_{i j}\left(E_{i j}+E_{j i}\right) .
$$

Then, we can show that $A=\sum_{i=1}^{n} \sum_{j>i} \sigma_{i j}\left(E_{i j}+E_{j i}\right)=$ $G_{\sigma}=G_{g(\theta)}$ by Definition 2.2 Moreover, $g(\theta)$ is an aggregated Markov process, since $\theta$ is a Markov process. Hence, we can rewrite $\Sigma$ as the aggregated Markov jump linear system $\dot{p}(t)=\left(B G_{g(\theta(t))}-D\right) p(t)$. The state transformation $x \mapsto B^{-1 / 2} x$, where $B^{-1 / 2}=\operatorname{diag}\left(\beta_{1}^{-1 / 2}, \ldots, \beta_{n}^{-1 / 2}\right)$, shows that almost sure exponential stability of this system is equivalent to almost sure exponential stability of the following aggregated Markov jump linear system:

$$
\Sigma_{2}: \dot{p}(t)=\left(B^{1 / 2} G_{g(\theta(t))} B^{1 / 2}-D\right) p(t) .
$$

To prove almost sure exponential stability of $\Sigma_{2}$, by Lemma 5.1, it is sufficient to prove that $E\left[\eta\left(B^{1 / 2} G_{g(\pi)} B^{1 / 2}-D\right)\right]<0$, where we have used the fact that $\eta(A)=\mu(A)$ for a symmetric matrix $A$. On the other hand, in the same way as we did in the proof 
of Proposition 5.2, we can prove that random matrices $B^{1 / 2} G_{g(\pi)} B^{1 / 2}-D$ and $M_{2}$ have the same probability distribution. Therefore, condition (32) is sufficient to guarantee almost sure exponential stability of $\Sigma_{2}$ and hence of $\Sigma$. Moreover, from the above argument, it is straightforward to see that the decay rate of the almost sure exponential stability is greater than or equal to $-E\left[\eta\left(M_{2}\right)\right]$. This completes the proof of the proposition.

Let us prove Theorem 3.4 .

Proof of Theorem 3.4 Let $\lambda_{2}=\eta(B \bar{A}-D)$ and define

$$
\begin{aligned}
X & =M_{2}-\lambda_{2} I-\kappa_{\bar{\beta}, \Delta_{2}}^{-1}(1) I \\
& =\left(-\lambda_{2} I-D-\kappa_{\bar{\beta}, \Delta_{2}}^{-1}(1) I\right)+\sum_{i=1}^{n} \sum_{j>i} X_{i j},
\end{aligned}
$$

where $X_{i j}=\sqrt{\beta_{i} \beta_{j}}\left(E_{i j}+E_{j i}\right) h_{i j}$. We now apply Proposition 5.3 to the random matrix $X$, for which we choose $C=\vec{\beta}$. Also, in the same way as we derived (28), we can show $v^{2}=\Delta_{2}$. Since $\eta(E[X])=-\kappa_{\bar{\beta}, \Delta_{2}}^{-1}(1)$, from 26] we obtain $\operatorname{Pr}\left(\eta(X)>-\kappa_{\bar{\beta}, \Delta_{2}}^{-1}(1)+s\right) \leq \kappa_{\bar{\beta}, \Delta_{2}}(s)$. Then, in the same way as we derived (30), we can show $E[\eta(X)] \leq-\kappa_{\bar{\beta}, \Delta_{2}}^{-1}(1)+s+\left(c_{2}-\lambda_{2}\right) \kappa_{\bar{\beta}, \Delta_{2}}(s)$, which implies $E\left[\eta\left(M_{2}\right)\right] \leq \lambda_{2}\left(1-\kappa_{\bar{\beta}, \Delta_{2}}(s)\right)+s+c_{2} \kappa_{\bar{\beta}, \Delta_{2}}(s)$. Using this inequality and Proposition 5.7, in the same way as we did in the proof of Theorem 3.2. we can show that condition (11) in the theorem is indeed sufficient for almost sure exponential stability of the disease-free equilibrium of $\Sigma$ with decay rate greater than or equal to (12).

Finally, we give an outline of the proof of Theorem 3.5 below.

Proof of Theorem 3.5 Consider an irreducible AMEI random graph process $\mathcal{G}$. Assume homogeneous spreading and recovery rates, $\beta_{i}=\beta>0$ and $\delta_{i}=\delta>0$ for all $i$. In the same way as we did in the proofs of Propositions 5.2 and 5.7. we can show that if $\eta\left(E\left[M_{3}\right]\right)<\delta / \beta$ for $M_{3}=\sum_{i=1}^{n} \sum_{j>i}\left(E_{i j}+E_{j i}\right) h_{i j}$, then the disease-free equilibrium of $\Sigma$ is almost surely exponentially stable with decay rate greater than or equal to $(\delta / \beta)-\eta\left(E\left[M_{3}\right]\right)$. Let $\lambda_{3}=\eta(\bar{A})$ and define $X=M_{3}-\lambda_{3} I-\kappa_{1, \Delta_{3}}^{-1}(1)$. Applying Proposition 5.3 to the random matrix $X$, we obtain

$$
E[\eta(X)] \leq-\kappa_{1, \Delta_{3}}^{-1}(1)+s+\left(c_{3}-\lambda_{3}\right) \kappa_{1, \Delta_{3}}(s) .
$$

Therefore, $E\left[\eta\left(M_{3}\right)\right] \leq \lambda_{3}+s+\left(c_{3}-\lambda_{3}\right) \kappa_{1, \Delta_{3}}(s)$. Using this inequality and the assumption (15), we can actually show $\eta\left(E\left[M_{3}\right]\right)<\delta / \beta$ and therefore the almost sure exponential stability of $\Sigma$ in the same way as the proofs of Theorems 3.2 and 3.4 We omit the details of the derivation of the lower bound (19) of the decay rate. Also, it is straightforward to show that 16 implies $\xi_{H}<1$. This completes the proof of the theorem.

\subsection{Proof of Theorem 3.10}

In this subsection, we give the proof of Theorem 3.10 for spreading processes running in discrete-time. In order to prove this theorem, we need to recall some facts regarding the stability of switched linear systems. Let $\sigma=f(\theta)$ be an aggregated Markov chain defined by the mapping $f: \Lambda \rightarrow$ $\Gamma$ and a Markov chain $\sigma$ with state space $\Lambda$. Let us define a state matrix $A_{\gamma} \in \mathbb{R}^{n \times n}$ for each $\gamma \in \Gamma$. A discrete-time
aggregated-Markov jump linear system is described by the stochastic difference equation

$$
x(k+1)=A_{f(\theta(k))} x,
$$

where $x(0)=x_{0}$ and $\theta(0)=\theta_{0}$. We say that the system in (34) is almost surely exponentially stable if there exists $\lambda>0$ such that $\operatorname{Pr}\left(\lim \sup _{k \rightarrow \infty} k^{-1} \log \|x(k)\| \leq-\lambda\right)=1$ for all $x_{0}$ and $\theta_{0}$. The supremum of $\lambda$ satisfying the above condition is called the decay rate. To prove Theorem 3.10, we need the following criterion for almost sure exponential stability of aggregated-Markov jump linear systems in discrete-time.

Lemma 5.8. Consider an irreducible Markov chain $\theta$ and denote its unique stationary distribution by $\pi$. Assume that $A_{\gamma}$ is nonnegative and symmetric for every $\gamma \in \Gamma$. if $E\left[\log \left(\eta\left(A_{f(\pi)}\right)\right)\right]<0$, then the discrete-time aggregated Markov jump linear system in (34) is almost surely exponentially stable with decay rate greater than or equal to $-E\left[\log \left(\eta\left(A_{f(\pi)}\right)\right)\right]$.

Proof: Let $\|A\|$ denote the maximum singular value of a matrix $A$. Since $\eta(A)=\|A\|$ for a nonnegative and symmetric matrix $A$, it is sufficient to show that $E\left[\log \left\|A_{f(\pi)}\right\|\right]<0$ implies almost sure exponential stability of the aggregated Markov jump linear system (34) with decay rate smaller than or equal to $-E\left[\log \left\|A_{f(\pi)}\right\|\right]$. This claim is known to be true if $f$ is the identity mapping, i.e., if $f(\theta)$ itself is a Markov chain [51, Proposition 2.1]. The proof for the general case where $f(\theta)$ is not necessarily a Markov chain has the same structure as the proof of Lemma 5.1. We omit the details.

Using this lemma, we prove the next proposition.

Proposition 5.9. Consider an irreducible and aperiodic AMEI random graph process $\mathcal{G}$ in discrete-time. Let $h_{i j}$ ( $1 \leq i<j \leq n$ ) be independent Bernoulli random variables with mean $\bar{A}_{i j}$. Define the random matrix

$$
M_{4}=I-D+\sum_{i=1}^{n} \sum_{j>i} \sqrt{\beta_{i} \beta_{j}}\left(E_{i j}+E_{j i}\right) h_{i j} .
$$

If

$$
E\left[\log \left(\eta\left(M_{4}\right)\right)\right]<0,
$$

then the disease-free equilibrium of $\Sigma_{d}$ is almost surely exponentially stable with decay rate greater than or equal to $-E\left[\log \left(\eta\left(M_{4}\right)\right)\right]$.

Proof: For each $k \geq 0$, let $A(k)$ denote the adjacency matrix of $\mathcal{G}(k)$. Define the product process $\theta$ on the state space $\Lambda$ in the same way as in the proof of Proposition 5.7 For $\gamma=\left(\gamma_{i j}\right)_{i, j} \in\{0,1\}^{n(n-1) / 2}$, consider the matrix $G_{\gamma}$ defined in (33). Then, in the same way as in the proof of Proposition 5.7, we can show $A(k)=G_{g(\theta(k))}$. Therefore $\Sigma$ is equivalent to the following discrete-time aggregated Markov jump linear system $p(k+1)=\left(B G_{g(\theta(k))}+I-\right.$ $D) p(k)$. Furthermore, the state transformation $x \mapsto B^{-1 / 2} x$ shows that almost sure exponential stability of this system is equivalent to that of the following aggregated-Markov jump linear system

$$
\Sigma_{4}: p(k+1)=\left(B^{1 / 2} G_{g(\theta(k))} B^{1 / 2}+I-D\right) p(k) .
$$

Since the matrix $B^{1 / 2} G_{g(\theta(k))} B^{1 / 2}+I-D$ is nonnegative and symmetric, by Lemma 5.8, the system $\Sigma_{4}$ is almost 
surely stable if $E\left[\log \left(\eta\left(B^{1 / 2} G_{g(\pi)} B^{1 / 2}+I-D\right)\right)\right]<0$, where $\pi$ denotes the stationary distribution of the irreducible and aperiodic Markov chain $\theta$. On the other hand, in the same way as in the proof of Proposition 5.7 we can show that the random matrices $B^{1 / 2} G_{g(\pi)} B^{1 / 2}+I-D$ and $M_{4}$ have the same probability distribution. Therefore, condition (35) is indeed sufficient for almost sure exponential stability of $\Sigma_{4}$, which implies almost sure exponential stability of the disease-free equilibrium of $\Sigma_{d}$ as we observed above. Also, from the above discussion, it is easy to verify that $-E\left[\log \left(\eta\left(M_{4}\right)\right)\right]$ gives a lower bound on the decay rate of stability.

We are now in condition to prove Theorem 3.10

Proof of Theorem 3.10 Applying Proposition 5.3 to the random matrix $M_{4}$, we obtain the inequality $\operatorname{Pr}\left(\eta\left(M_{4}\right)>\eta\left(E\left[M_{4}\right]\right)+s\right) \leq \kappa_{\bar{\beta}, \Delta_{2}}(s)$. Since $\lambda_{4}=$ $\eta(B \bar{A}+I-D)=\eta\left(E\left[M_{4}\right]\right)$, this inequality implies that

$$
\operatorname{Pr}\left(\frac{\eta\left(M_{4}\right)}{\lambda_{4}}>1+\frac{s}{\lambda_{4}}\right) \leq \kappa_{\bar{\beta}, \Delta_{2}}(s)
$$

for every $s \geq 0$. Therefore, in the same way as we derived (30), we can show that

$$
E\left[\log \frac{\eta\left(M_{4}\right)}{\lambda_{4}}\right] \leq \log \left(1+\frac{s}{\lambda_{4}}\right)+\kappa_{\bar{\beta}, \Delta_{2}}(s) \log \frac{\eta\left(M_{\max }\right)}{\lambda_{4}}
$$

and, hence,

$$
E\left[\log \left(\eta\left(M_{4}\right)\right)\right] \leq \log \left(\lambda_{4}+s\right)+\kappa_{\bar{\beta}, \Delta_{2}}(s) \log \frac{\eta\left(M_{\max }\right)}{\lambda_{4}} .
$$

Therefore, if there exists $s \in\left[0,1-\lambda_{4}\right]$ such that the right hand side of this inequality is negative, then disease-free equilibrium of $\Sigma_{d}$ is almost surely exponentially stable by Proposition 5.9. A simple algebra shows that the existence of such $s \geq 0$ is equivalent to (18). Notice that we do not need to consider any $s$ larger than $1-\lambda_{4}$ because, for such $s$, the right hand side of 36 is positive. Moreover, the upper bound (19) of the decay rate immediately follows from (36). This completes the proof of the theorem.

\section{CONCLUSION AND DISCUSSION}

In this paper, we have analyzed the dynamics of spreading processes taking place over time-varying networks. First, we have proposed the family of aggregated-Markovian random graph processes as a flexible and analytically tractable dynamic random graph able to replicate, with arbitrary accuracy, any distribution of inter-switching times. We have then studied spreading processes in aggregated-Markovian random graph processes and derived conditions to guarantee that the disease-free equilibrium is almost surely exponentially stable. A direct analysis based on Itô's formula for jump processes results in stability conditions in terms of the eigenvalues of a matrix whose size grows exponentially with the number of edges in the network. Using tools from random graph theory, we have derived alternative stability conditions in terms of the eigenvalues of a matrix whose size grows linearly with the number of nodes in the graph. Based on our theoretical results, we have shown that (i) aggregated static networks approximate the epidemic threshold more accurately as the number of nodes in the network grows, and (ii) aggregated static networks provide a better approximation as we reduce the degree of temporal variability in the random graph process.

A possible direction for future research is containment of epidemic outbreaks over time-varying networks. Although several optimization frameworks have been recently proposed in the literature to find the cost-optimal allocation of medical resources to prevent epidemic outbreaks in static networks [11], [12], [13], [14], these results cannot be readily applied to the case of time-varying networks.

\section{REFERENCES}

[1] K. Lerman and R. Ghosh, "Information contagion: An empirical study of the spread of news on Digg and Twitter social networks," in Proceedings of the Fourth International AAAI Conference on Weblogs and Social Media, 2010, pp. 90-97.

[2] M. Garetto, W. Gong, and D. Towsley, "Modeling malware spreading dynamics," in IEEE INFOCOM 2003. Twenty-second Annual Joint Conference of the IEEE Computer and Communications Societies, vol. 3, 2003, pp. 1869-1879.

[3] M. Tizzoni, P. Bajardi, C. Poletto, J. J. Ramasco, D. Balcan, B. Gonçalves, N. Perra, V. Colizza, and A. Vespignani, "Real-time numerical forecast of global epidemic spreading: case study of 2009 A/H1N1pdm." BMC medicine, vol. 10, p. 165, 2012.

[4] R. Pastor-Satorras, C. Castellano, P. Van Mieghem, and A. Vespignani, "Epidemic processes in complex networks," Reviews of Modern Physics, vol. 87, pp. 925-979, 2015.

[5] C. Nowzari, V. M. Preciado, and G. J. Pappas, "Analysis and control of epidemics: A survey of spreading processes on complex networks," IEEE Control Systems Magazine (accepted), 2015.

[6] H. J. Ahn and B. Hassibi, "On the mixing time of the SIS Markov chain model for epidemic spread," in 53rd IEEE Conference on Decision and Control, 2014, pp. 6221-6227.

[7] A. Ganesh, L. Massoulié, and D. Towsley, "The effect of network topology on the spread of epidemics," in Proceedings - IEEE INFOCOM, vol. 2, 2005, pp. 1455-1466.

[8] D. Chakrabarti, Y. Wang, C. Wang, J. Leskovec, and C. Faloutsos, "Epidemic thresholds in real networks," ACM Transactions on Information and System Security, vol. 10, 2008.

[9] P. Van Mieghem, J. Omic, and R. Kooij, "Virus spread in networks," IEEE/ACM Transactions on Networking, vol. 17, pp. 1-14, 2009.

[10] F. Darabi Sahneh, C. Scoglio, and P. Van Mieghem, "Generalized epidemic mean-field model for spreading processes over multilayer complex networks," IEEE/ACM Transactions on Networking, vol. 21, pp. 1609-1620, 2013.

[11] Y. Wan, S. Roy, and A. Saberi, "Designing spatially heterogeneous strategies for control of virus spread." IET systems biology, vol. 2, pp. 184-201, 2008.

[12] V. M. Preciado, M. Zargham, C. Enyioha, A. Jadbabaie, and G. Pappas, "Optimal vaccine allocation to control epidemic outbreaks in arbitrary networks," in 52nd IEEE Conference on Decision and Control, 2013, pp. 7486-7491.

[13] V. M. Preciado, M. Zargham, C. Enyioha, A. Jadbabaie, and G. J. Pappas, "Optimal resource allocation for network protection against spreading processes," IEEE Transactions on Control of Network Systems, vol. 1, pp. 99-108, 2014.

[14] K. Drakopoulos, A. Ozdaglar, and J. Tsitsiklis, "An efficient curing policy for epidemics on graphs," IEEE Transactions on Network Science and Engineering, vol. 1, pp. 67-75, 2014.

[15] P. Holme and J. Saramäki, "Temporal networks," Physics Reports, vol. 519, pp. 97-125, 2012.

[16] N. Fefferman and $\mathrm{K} . \mathrm{Ng}$, "How disease models in static networks can fail to approximate disease in dynamic networks," Physical Review E, vol. 76, p. 031919, 2007.

[17] N. Masuda and P. Holme, "Predicting and controlling infectious disease epidemics using temporal networks." F1000prime reports, vol. 5:6, 2013.

[18] A. Vazquez, B. Rácz, A. Lukács, and A.-L. Barabási, "Impact of non-Poissonian activity patterns on spreading processes," Physical Review Letters, vol. 98, p. 158702, 2007.

[19] M. Karsai, M. Kivelä, R. K. Pan, K. Kaski, J. Kertész, A.-L. Barabási, and J. Saramäki, "Small but slow world: How network topology and burstiness slow down spreading," Physical Review E, vol. 83, p. 025102, 2011. 
[20] P. Holme and F. Liljeros, "Birth and death of links control disease spreading in empirical contact networks." Scientific reports, vol. 4, p. $4999,2014$.

[21] C. L. Vestergaard, M. Génois, and A. Barrat, "How memory generates heterogeneous dynamics in temporal networks," Physical Review E, vol. 90, p. 042805, 2014.

[22] E. Volz and L. A. Meyers, "Epidemic thresholds in dynamic contact networks." Journal of the Royal Society, Interface / the Royal Society, vol. 6, pp. 233-241, 2009.

[23] Y. Schwarzkopf, A. Rákos, and D. Mukamel, "Epidemic spreading in evolving networks," Physical Review E, vol. 82, p. 036112, 2010.

[24] N. Perra, A. Baronchelli, D. Mocanu, B. Gonçalves, R. PastorSatorras, and A. Vespignani, "Random walks and search in timevarying networks," Physical Review Letters, vol. 109, p. 238701, 2012.

[25] N. Perra, B. Gonçalves, R. Pastor-Satorras, and A. Vespignani, "Activity driven modeling of time varying networks." Scientific reports, vol. 2:469, 2012.

[26] M. Karsai, N. Perra, and A. Vespignani, "Time varying networks and the weakness of strong ties." Scientific reports, vol. 4, p. 4001, 2014.

[27] A. E. Clementi, C. Macci, A. Monti, F. Pasquale, and R. Silvestri, "Flooding time in edge-Markovian dynamic graphs," in Proceedings of the twenty-seventh ACM symposium on Principles of distributed computing - PODC '08, 2008, pp. 213-222.

[28] I. Z. Kiss, L. Berthouze, T. J. Taylor, and P. L. Simon, “Modelling approaches for simple dynamic networks and applications to disease transmission models," Proceedings of the Royal Society A Mathematical, Physical and Engineering Sciences, vol. 468, pp. 13321355, 2012.

[29] J. Whitbeck, V. Conan, and M. D. de Amorim, "Performance of opportunistic epidemic routing on edge-Markovian dynamic graphs," IEEE Transactions on Communications, vol. 59, pp. 12591263, 2011.

[30] L. Maggi and F. De Pellegrini, "Not always sparse: Flooding time in partially connected mobile ad hoc networks," 2014 12th International Symposium on Modeling and Optimization in Mobile, Ad Hoc, and Wireless Networks, WiOpt 2014, pp. 413-420, 2014.

[31] H. Baumann, P. Crescenzi, and P. Fraigniaud, "Parsimonious flooding in dynamic graphs," Proceedings of the 28th ACM symposium on Principles of distributed computing - PODC '09, p. 260, 2009.

[32] M. Taylor, T. J. Taylor, and I. Z. Kiss, "Epidemic threshold and control in a dynamic network," Physical Review E, vol. 85, p. 016103, 2012.

[33] C. Cattuto, W. van den Broeck, A. Barrat, V. Colizza, J. F. Pinton, and A. Vespignani, "Dynamics of person-to-person interactions from distributed RFID sensor networks," PLOS ONE, vol. 5, pp. $1-9,2010$.

[34] J. Stehlé, N. Voirin, A. Barrat, C. Cattuto, L. Isella, J. F. Pinton, M. Quaggiotto, W. van den Broeck, C. Régis, B. Lina, and P. Vanhems, "High-resolution measurements of face-to-face contact patterns in a primary school," PLoS ONE, vol. 6, 2011.

[35] J. Stehlé, N. Voirin, A. Barrat, C. Cattuto, V. Colizza, L. Isella, C. Régis, J.-F. Pinton, N. Khanafer, W. Van den Broeck, and P. Vanhems, "Simulation of an SEIR infectious disease model on the dynamic contact network of conference attendees." BMC medicine, vol. 9, p. 87, 2011.

[36] D. R. Fredkin and J. A. Rice, "On aggregated Markov processes," Journal of Applied Probability, vol. 23, pp. 208-214, 1986.

[37] M. A. Rami, V. S. Bokharaie, O. Mason, and F. R. Wirth, "Stability criteria for SIS epidemiological models under switching policies," Discrete and Continuous Dynamical Systems - Series B, vol. 19, pp. 2865-2887, 2014.

[38] R. W. Brockett, "Stochastic Control," 2009. [Online]. Available: http://www.eeci-institute.eu/pdf/M015/RogersStochastic.pdf

[39] F. Chung, L. Lu, and V. Vu, "Spectra of random graphs with given expected degrees," Proceedings of the National Academy of Sciences of the United States of America, vol. 100, pp. 6313-6318, 2003.

[40] C. Desoer and H. Haneda, "The measure of a matrix as a tool to analyze computer algorithms for circuit analysis," IEEE Transactions on Circuit Theory, vol. 19, pp. 480-486, 1972.

[41] E. Çinlar, "Exceptional paper-Markov renewal theory: A survey," Management Science, vol. 21, pp. 727-752, 1975.

[42] R. Schassberger, "On the waiting time in the queuing system GI/G/1," The Annals of Mathematical Statistics, vol. 41, pp. 182$187,1970$.
[43] C. Lanczos, "An iteration method for the solution of the eigenvalue problem of linear differential and integral operators," Journal of Research of the National Bureau of Standards, vol. 45, pp. 255-282, 1950.

[44] J. Saramäki and K. Kaski, "Modelling development of epidemics with dynamic small-world networks." Journal of theoretical biology, vol. 234, pp. 413-21, 2005.

[45] L. Erdős and A. Rényi, "On random graphs. I," Publicationes Mathematicae, vol. 6, pp. 290-297, 1959.

[46] E. Cator and P. Van Mieghem, "Susceptible-infected-susceptible epidemics on the complete graph and the star graph: Exact analysis," Physical Review E, vol. 87, p. 012811, 2013.

[47] O. L. V. Costa, M. D. Fragoso, and M. G. Todorov, Continuous-time Markov Jump Linear Systems. Springer, 2013.

[48] Y. Fang and K. Loparo, "Stabilization of continuous-time jump linear systems," IEEE Transactions on Automatic Control, vol. 47, pp. 1590-1603, 2002.

[49] F. Chung and M. Radcliffe, "On the spectra of general random graphs," The Electronic Journal of Combinatorics, vol. 18, \#P215, 2011.

[50] N. K. Son and D. Hinrichsen, "Robust stability of positive continuous time systems," Numerical Functional Analysis and Optimization, vol. 17, pp. 649-659, 1996.

[51] P. Bolzern, "On almost sure stability of discrete-time Markov jump linear systems," in 43rd IEEE Conference on Decision and Control, 2004, pp. 3204-3208.

\section{ACKNOWLEDGMENTS}

This work was supported in part by the NSF under grants CNS-1302222 and IIS-1447470. A part of this research was performed while the first author was visiting the Center for BioCybernetics and Intelligent Systems at Texas Tech University. He would like to thank Professor Bijoy Ghosh for his hospitality during this visit.

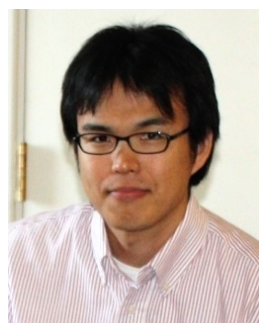

Masaki Ogura received his B.Sc. degree in engineering and M.Sc. degree in informatics from Kyoto University, Japan, in 2007 and 2009, respectively, and his Ph.D. degree in mathematics from Texas Tech University in 2014. He is currently a Postdoctoral Researcher in the Department of Electrical and Systems Engineering at the University of Pennsylvania. His research interest includes dynamical systems on timevarying networks, switched linear systems, and stochastic processes.

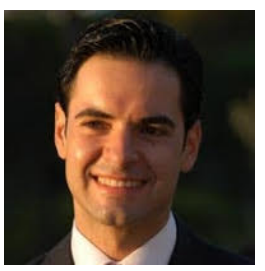

Victor M. Preciado received his Ph.D. degree in Electrical Engineering and Computer Science from the Massachusetts Institute of Technology in 2008. He is currently the Raj and Neera Singh Assistant Professor of Electrical and Systems Engineering at the University of Pennsylvania. $\mathrm{He}$ is a member of the Networked and Social Systems Engineering (NETS) program and the Warren Center for Network and Data Sciences. His research interests include network science, dynamic systems, control theory, and convex optimization with applications in socio-technical systems, technological infrastructure, and biological networks. 